\title{
Beyond abstract film: constructivist digital time
}

\author{
Ernest Edmonds \\ Creativity and Cognition Studios \\ University of Technology, Sydney \\ P.O. Box 123 Broadway \\ NSW 2007 \\ Australia \\ ernest@ernestedmonds.com
}

\begin{abstract}
The paper reviews aspects of abstract films and the notions of time that occur in them. A series of developments by the author in making various generative digital abstract, or concrete, works are described and compared to film. The generation of the time element of the works described is integral with the generation of images. It is shown how different approaches to dealing with time in the digital context have emerged. In particular, an integrated constructivist approach has built from concepts in abstract film to go beyond cinema in a way that makes significant use of digital media
\end{abstract}

Time. Concrete art. Abstract art. Digital. Film. Video. Generative.

\section{INTRODUCTION}

This paper is concerned with abstract arts and in particular the links between abstract digital art and abstract film. However, the use of the word 'abstract' in art is a little odd. In ordinary language the noun 'abstract' refers to a reduced representation of something. An 'abstraction' would similarly normally refer to a form of representation. The art use of the term seems to come from a concern with representational figurative paintings, which might be thought of as abstract representations of landscapes or people. By contrast the term was applied to art that, in fact, is an abstract of nothing at all. The movement towards abstract art, from Cézanne, through Cubism and to Kandinsky, Malevich and Mondrian, for example, was in a direction of increasingly sparse or distant abstraction from the subjects of figurative painting. Once the work ceased to reference external reality at all, and hence ceased to be an abstraction, the label abstract somehow, inappropriately, stuck.

Lawrence Alloway defined useful ways of handling the problem: 'I propose to use abstract meaning to draw out of or draw away from. Figurative paintings and landscapes will be said to have been abstracted from figures and landscapes. The word concrete will be used for works of art in which a process of abstraction is not perceptible.... I am aware of the logical objections to the term nonfigurative but ... It is used here as an exclusive term for the whole field.' (Alloway, 1954).
Conny Dietzschold elaborates on concrete art, 'this was the area of modern art which began in Europe around 1910, to detach itself entirely from the reproduction of the object ... the term "concrete" is to be distinguished from "abstract" - the latter denoting art which does not relinquish its relationship to reality. ... by definition it is the autonomy of pictorial means which constitutes Concrete Art, their insistence that the pictorial world is a world in and of itself, founded on surface, space, line, colour, light, light/dark contrasts and movement.' (Dietzschold, 2005).

Concrete art is concerned very directly with reality. It is concerned with the reality of the art object and our perception of it. Through challenging perception and making concrete art that addresses the very substance of surface, colour, movement and so on, the artist is extending our language and hence constructing new realities. If a work changes in time, as a film does, for example, then the properties of the transitions in time are part of the concrete reality of that work.

A significant proportion of digital art is time-based. It may interact with the environment and/or the audience or it may simply change over time in the manner of film. In any case, time is often an important factor in digital works. Strangely, perhaps, in discussing digital art we seem to put more emphasis on, for example, the graphical displays, the sounds and the modes of interaction than time itself. Music is, of course, a classic timebased medium and film also has a serious history. In both cases, time receives significant attention. 
Having discussed the concrete/abstract issue we can continue to use the term 'abstract' in what follows in its normal art meaning but being clear that the material reality of the art object rather than some abstracted representation of the world is what the paper is concerned with.

\section{ABSTRACT FILM}

Various time-based media have been important in leading to current developments in digital art. A concern for the media themselves and their formal concrete properties has a significant history that is relevant to current developments. Looking back over his work in film, for example, Hans Richter characterised his practice in terms of the organisation of abstract forms and said this was about 'a universal language, which is what abstract art should be about' (Foster, 1998, p. 172). This was not a new position to take. Indeed, Cézanne said, 'The technique of any art consists of a language and a logic' (Larguier, 2001). This concern for the concrete in art was not confined to painting and Richter was not the only interested filmmaker. For example, in discussing Vertov's movies, Malevich said, 'Here, ... the elements are not shackled together in a whole in order to convey the gossip of existence ... He shows the movement itself, the dynamics whose force has been previously overshadowed by the cigarette-holder...' (Malevich, 2002). In fact, Vertov 'wanted not only to demonstrate the material and constructed nature of film, but also the reality constructed with it.' (Weibel, 1979).

Malcolm Le Grice published his book Abstract Film and Beyond in 1977 (Le Grice, 1977). This was an important and thorough review of developments up to that time of film as a concrete medium. The concerns covered in this book were not those of film as a vehicle for story telling but with the film medium itself. Abstract film began with a nonrepresentational position, which was drawn largely from painting, rather than theatre. This distinction was discussed in the early days, for example, in the writings of Malevich (Bulkagoa, 2002). As one might expect from his painting, he strongly supported the idea of abstract film (without calling it that).

The first abstract films date from 1910 to 1912. They were made by Bruno Corra and Arnaldo Ginna. Cora described their exploration as a series of artistic experiments that had quite varied levels of success: '.. after the entire theory had been minutely established, we decided to make a serious attempt to create a music of colors. We immediately began to think of the instruments, which perhaps did not exist,.... We turned our thoughts to cinematography, and it seemed to us that this medium, slightly modified, would give excellent results, since its light potency was the strongest one could desire. The other problem concerning the need to have hundreds of colors at our disposition was also resolved, since, by exploiting the phenomenon of the persistence of an image on the retina, we would indeed have been able to make many colors merge, in our eye, into a single hue. To achieve this it was sufficient to pass all the component colors in front of the lens in less than a tenth of a second. In this way with a simple cinematographic instrument, with a machine of small dimensions, we would have obtained the innumerable and extremely powerful effects of large musical orchestras, the true chromatic symphony...' (Cora, 1912).

Peter Kubelka's Arnulf Rainer from 1960 demonstrated abstract film in its most pure concrete form (Kubelka, 1960). It contains only pure white and pure black frames. It is '...a constructivist work, a visual music that develops a pattern of relationships between the periods of black screen and those of white...' Kubelka treated time in the manner of music and related it to constructivist art.

The relationship of abstract film to music seems obvious and it is not suprising to read Cora's and Kubelka's descriptions using musical terms. However, the concern moved on, as Le Grice described it, to explore the concrete medium itself in its own terms. Thus film has very particular properties. For example, it consists a fixed number of frames that are shown in any one second. Altogether, a significant issue in abstract film is the manipulation of time, including the relationship between time in the recording (or filming) process to time in projection. These can be quite different and changes between one and the other are often used as a significant feature of a film's properties.

\section{FILM AND TECHNOLOGICAL INNOVATIONS}

Le Grice mentions a few computer generated abstract films that had by then been produced. These were made frame-by-frame, of course. One might say that the computer acted as a very fast animator, generating frames at whatever pace was convenient for recording, with the actual pace of the film realised at the time of projection.

As Le Grice published his book technological change was rife. From the introduction of Sony's Portapak in 1967, the new medium of video recording emerged. Whilst similar in many ways to film, its differences were such that the genre of 'video art' developed in a direction distinct from film. This genre has remained to this day, 
notwithstanding the extensive incorporation of video recording technologies into film making. At the same time, it is hard to see a feature film today that has not also used computer generated elements. The technologies have merged but, in terms of the concrete time-based arts, the distinctions still matter as are explored below.

Incorporating computers into artworks has extended the possibilities still further with the option, for example to make works that change for ever and that do not have to exist within a fixed time period.

\section{GENERATIVE ART AND TIME}

Generative art was first seen as algorithmic, i.e. art produced with the aid of a computer by programming it to follow some procedure that generated the art object. Today, such processes are most often associated with time-based art in which the generation of images is seen as a projection over time by the audience or viewer. Often, as in the author's work, the algorithmic element is dealt with by using declarative, rather than procedural, programming. The declarative description states what is required in terms of rules and it is left to the computer system to work out the order in which things should be done.

\section{GENERATIVE VIDEO ART}

The paper is concerned with the considerations of time in concrete, abstract, film making and with computer generated images and video recording from the early 1980 s and with the implications that remain today.

Between 1980 and 1985 the author developed a system for making time based abstract artworks that were generated by computer in real time (i.e. in the time employed for projection) and recorded directly onto videotape. The first completed piece was Fragments which lasted two hours and was shown as part of an art exhibition in London in 1985. See Figures 1,2,3,4. The images consisted of various arrangements of black and white squares as well as pure black and pure white frames. The key point about this work was the full incorporation of the time element into the generative process implemented within the computer. Time was a concrete part of the constructed work.

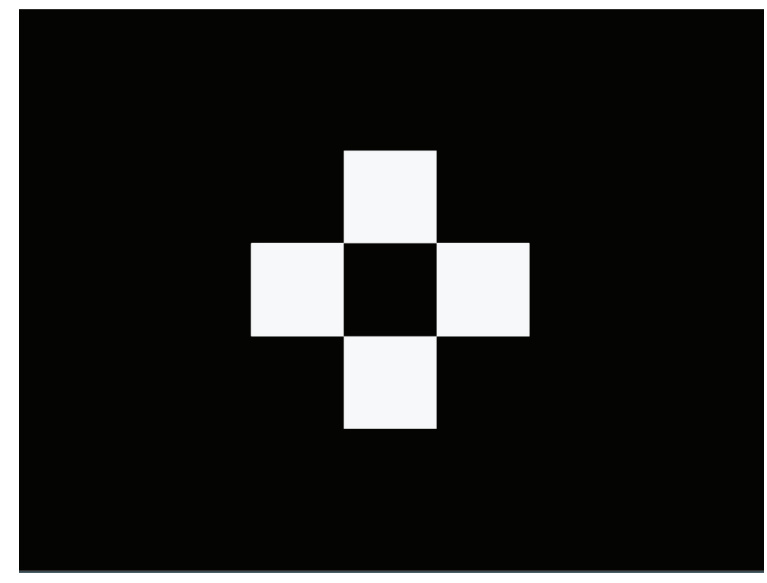

Figure 1: Still from Fragments

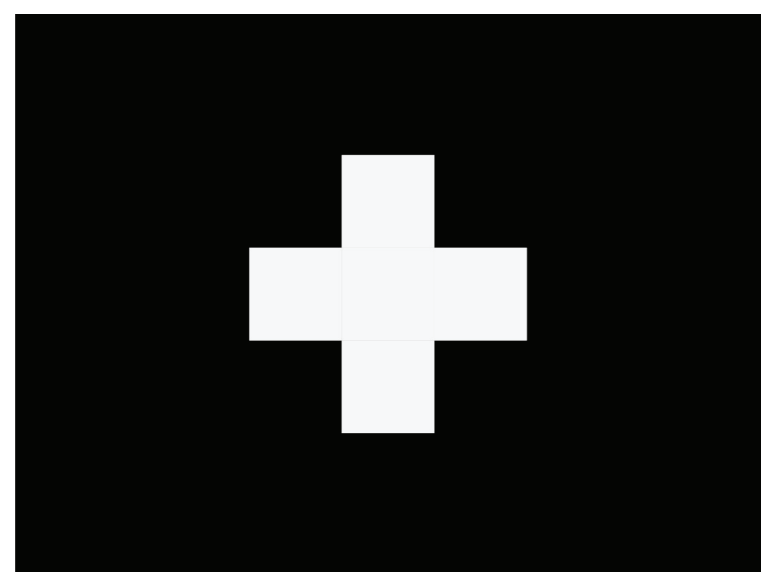

Figure 2: Still from Fragments

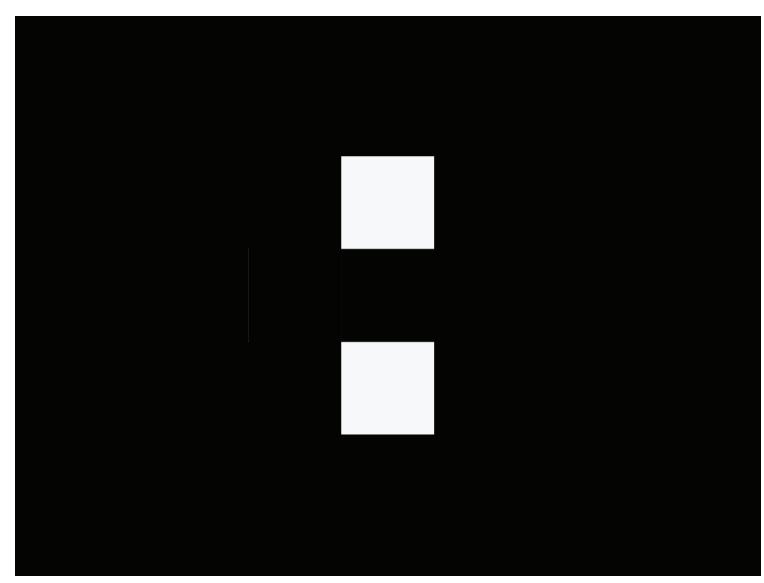

Figure 3: Still from Fragments 


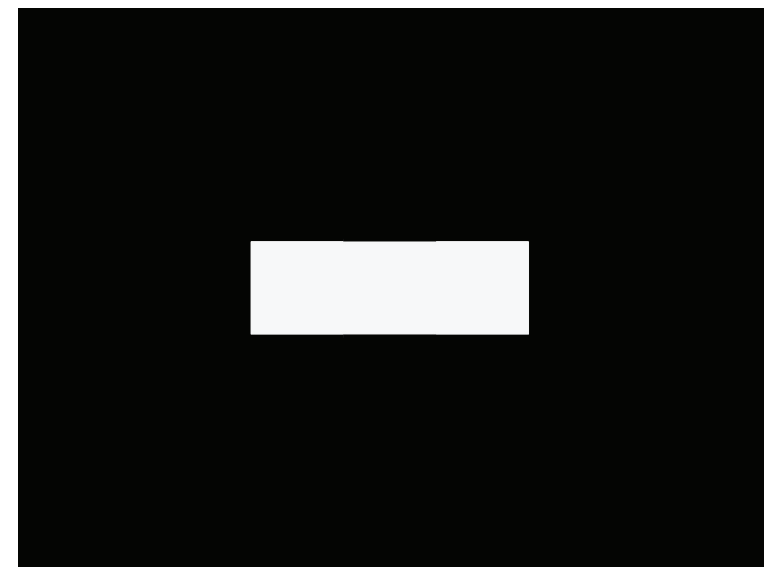

Figure 4: Still from Fragments

In animation, one can see the film as an ordered set of images, that may or may not be computer generated. Time is then imposed on the order by allocating a period for each images to be displayed and hence determine how many identical frames should be generated. In this sense, time is an independent aspect of the film. The timeline may be composed separatley from the image design, In addition the time in recording or filming need have no direct relationship with projection time.

In the approach used for Fragments, however, the generation of images and the timings are integrated. A generative computer program was used to define the work and produce it as computer output in real time. Rather than recording frames on film, the output from the computer was fed directly into a Umatic video recorder. In the computer program, a set of generative rules were defined that specified a search through a space of image possibilities. The space was a simple grid in which squares could either be black or white. The searching method is based on backtracking in logic programming (Edmonds, 1988). The time structures of the work are an integral part of the searching process.

In Fragments, and other work done at that time, both the images and the timing are determined by the generative rules as the computer system works through them. The totality of the work, therefore is completely implicit in the defining rules.

It was noted that Kulbelka said of his work Arnulf Rainer that it was '... a constructivist work, a visual music that develops a pattern of relationships ...'. 'Fragments' did exactly that, but because it was a computer generated work, as described above, the pattern of relationships over time were literally constructed in real time as the piece evolves. Another factor in this piece was that as there was no need to build it frame by frame it could be much longer than abstract films has typically been. It was shown in a gallery, rather than a cinema and people could come and go, watching just a part of it. It was interesting, however, that several interested artists were seen to watch the full two hours.

The images used in Fragments were deliberately simple because the complexity of change in time was felt to be sufficient. Additionally, the technology of colour screens was not reliable, and therefore no colour was used. Soon afterwards technology improved in that respect, with colour callibration becoming more available. A work that used a limited colour range was made and shown in Rotterdam in 1989. That work was called Sydney. See Figures 5,6,7. It lasted just 15 minutes. It was produced in exactly the same way as Fragments but with a more populated grid and, of course, generative rules for determining colour choices as well as the other aspects of the work. Thus Sydney was also a constructivist work with time, shape and colour relationships developed from a single set of generative rules.

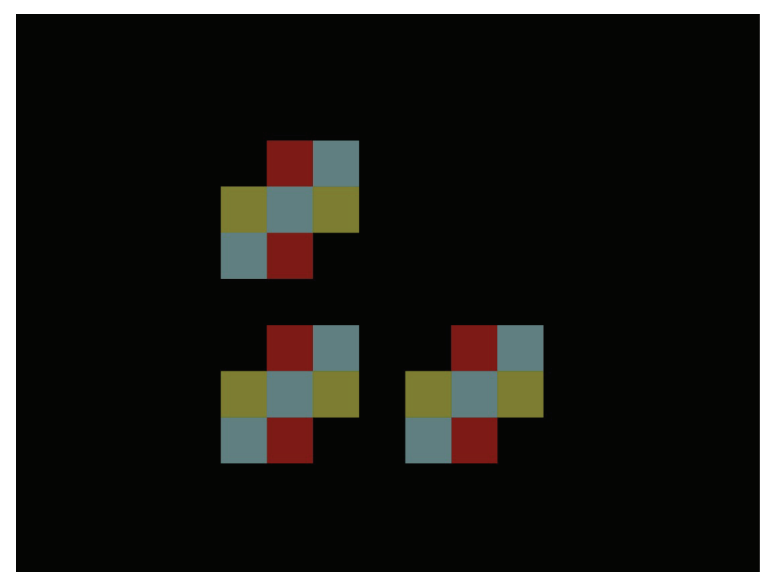

Figure 5: Still from Sydney

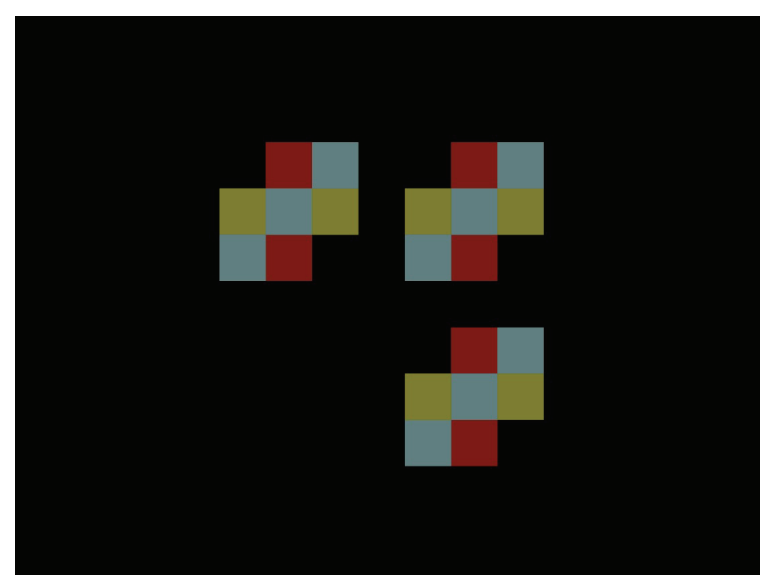

Figure 6: Still from Sydney 


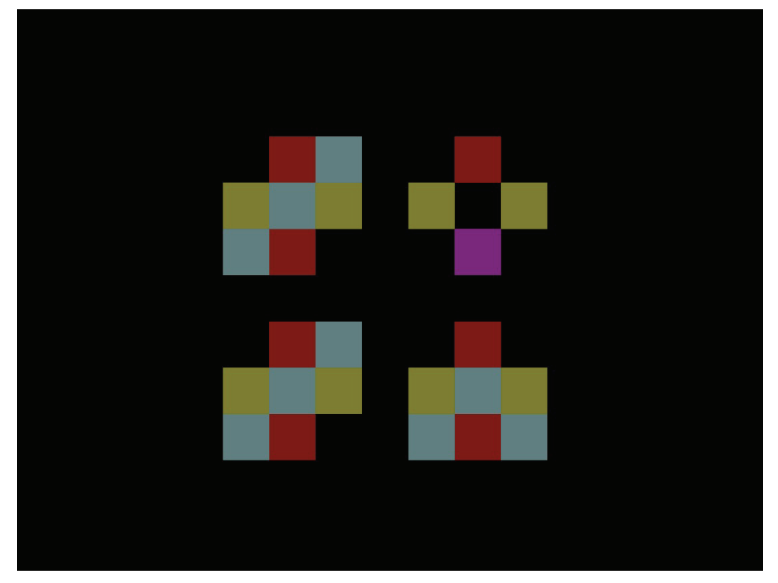

Figure 7: Still from Sydney

\section{CONSTRUCTIVE INTERACTION}

Today, of course, the technology allows for considerable more sophistication, but the same key concerns remain. More recent work has added input from image analysis systems and removed the notion of a fixed duration altogether.

Of course, the incorporation of the analysis of motion rules out the use of videotape, but by showing the works on high quality displays, suitably mounted, a further step can be taken in relation to time. The generative process may continue effectively for ever. As the rules are constantly being revised we have ever changing works that can exist over long time periods and, in that sense go beyond time, as used in film.

In a series of such works known as Shaping Form a camera is placed under the screen and the computer constantly analyses the movements of people in front of the work. Rather than leading to direct interaction, the computer system builds up a history of the movements and slowly modifies the generating rules being applied. In this way, the Shaping Form works are always changing, using new sets of colours and modifying the pace of change (Edmonds, 2007a).

As has been discussed elsewhere (Edmonds, 2007b), the processes of interacting with the world being used in artworks such as 'Shaping Form' do not fall into the more common category of gamelike interactive systems. The artwork changes its nature over long periods of time in response to events. It is influenced over time, one might say, rather than acting in a direct interaction mode.

\section{CONCLUSION}

The paper has reviewed aspects of abstract films and the notions of time that occur in them. The interest in the concrete reality of the film, rather than its narrative is the abstract aspect that is carried over into the digital works discussed. The specific role of time is emphasised. A series of developments by the author in making various generative digital abstract works have been described and compared to film. The generation of the time element of the works described is integrated with the generation of images. It has been shown how different approaches to dealing with time in the digital context have emerged. In particular, an integrated constructivist approach has developed from concepts in abstract film to go beyond such films in a way that makes significant use of the properties of digital media.

\section{REFERENCES}

Alloway, L. (1954) Nine Abstract Artists. Tiranti, London,

Bulgakowa, O. (ed) (2002) Kazimir Malevich: The White Rectangle, Writings on Film. Potemkin Press, San Francisco, CA.

Corra, B. (1912) Il pastore, il gregge e la zampogna. English translation, Abstract CinemaChromatic Music. In Apollini, U. (ed) (2009) Futurist Manifestos. Tate Publishing, London. Also at http://www.unknown.nu/futurism/abstract.html.

Dietzschold, C. (2005) Opening Speech: Minimal Approach...Concrete Tendencies. Tin Sheds Gallery, University of Sydney, April 2005.

Edmonds, E. (1988) Logic and time-based art practice. Leonardo, Electronic Art Supplemental issue, pp. 19-20.

Edmonds, E. (2007a) Shaping Form Series. In Jennings, O. (editor) Speculative Data and the Creative Imaginary. National Academy of Sciences, Washington DC. pp. 18-9.

Edmonds, E. (2007b). Reflections on the Nature of Interaction. CoDesign: International Journal of CoCreation in Design and the Arts. Vol. 3, Issue 3. pp. 139-143.

Foster, S. (ed) (1998) Hans Richter: Activism, Modernism and the Avant-Garde. MIT Press, Cambridge, MA.

Kubelka, P. (1960) Arnulf Rainer. YouTube version. http://www.youtube.com/watch?v=iw1DVtFAz64

Larguier, L. (2001) published this quotation 'collected by Cézanne's son without adding one line of my own.' Printed in English translation in Conversations with Cézanne, edited by Michael Doran, University of California Press, 2001, p. 17. 
Le Grice, M. (1977) Abstract Film and Beyond. Studio Vista, London.

Malevich, M. (2002) Pictorial Laws in Cinematic Problems. In Bulgakowa, O. Kazimir Malevich: The White Rectangle, Writings on Film. Potemkin

Press, San Francisco, CA. pp. 77-84.
Weibel, P. (1979) Eisenstein, Vertov and the Formal Film. In Film as Film, Hayward Gallery, London, pp. 47-51. 